\title{
artigo
}

Medeiros, A.C.T.; Lucena, N.C.; Barros, D.R.R.E.; Mélo, M.C.S.; Araújo, H.S.P.; Araújo, K.M.F.A.;

Experiência de ações educativas em saúde com idosos na atenção primária

\section{Experiência de ações educativas em saúde com idosos na atenção primária}

\author{
Experience of educational actions in health with elderly people in primary care \\ Experiencia de acciones educativas en salud con ancianos en atención primaria
}

\begin{abstract}
RESUMO
Objetivo: Relatar experiências de ações educativas em saúde com idosos da Atenção Primária à Saúde. Método: Estudo qualitativo, descritivo, tipo relato de experiência, realizado em uma Unidade Básica de Saúde, no município de Campina Grande-PB. Para o desenvolvimento das ações educativas, utilizou-se como estratégia a implementação da Caderneta de Saúde do Idoso desenvolvendo as temáticas apresentadas nela. Resultados: Por meio de ações educativas proporcionadas pelo projeto de extensão, possibilitou-se implementar a Caderneta na unidade conforme preconização do Ministério da Saúde, otimizando o processo de trabalho e promovendo uma avaliação integral da pessoa idosa. Importante enfatizar a construção contínua do conhecimento por meio de ações coletivas proporcionando melhor troca de conhecimentos. Conclusão: Espera-se que esta vivência, sensibilize a comunidade acadêmica quanto à importância das práticas educativas por meio da extensão universitária, proporcionando a troca de conhecimentos de forma coletiva, contribuindo para a construção pessoal e profissional de todos que dele participaram.

DESCRITORES: Saúde do idoso; Educação em saúde; Atenção Primária à Saúde; Enfermagem; Relações Comunidade-Instituição.
\end{abstract}

\section{ABSTRACT}

Objective: Report educational health actions experiences with elderly in Primary Health Care. Method: Qualitative, descriptive study of an experience report, performed in a Basic Health Unit, in the city of Campina Grande-PB. For the educational actions development, the implementation of the Health Handbook for the Elderly was used as a strategy, developing the themes in it. Results: Through educational actions provided by the extension project, it was possible to implement the Handbook in the unit as recommended by the Ministry of Health, optimizing the work process and a comprehensive assessment of the elderly. It is important to emphasize the continuous construction of knowledge through collective actions providing a better exchange of knowledge. Conclusion: It is expects that this experience will raise the awareness of the academic community regarding the importance of educational practices through university extension, providing collectively knowledge exchange, contributing to the personal and professional construction of the participants.

DESCRIPTORS: Health of the Elderly; Health Education; Primary Health Care; Nursing; Community-Institutional Relations.

\section{RESUMEN}

Objetivo: Informar experiencias de acciones educativas en salud con ancianos en Atención Primaria de Salud. Método: Estudio cualitativo, descriptivo, tipo de relato de experiencia, realizado en una Unidad Básica de Salud, en la ciudad de Campina Grande-PB. Para las acciones educativas, se desarrolló como estrategia la implementación del Manual de Salud para Personas Mayores, desarrollando temas presentados en la misma. Resultados: Mediante las acciones provistas por el proyecto de extensión, se logró implementar el Manual en la unidad según lo recomendado por el Ministerio de Salud, optimizando el proceso de trabajo y evaluación integral de los ancianos. Importante enfatizar la construcción continua de conocimiento mediante acciones colectivas que brinden mejor intercambio de conocimiento. Conclusión: Se espera que esta experiencia concientice la comunidad académica sobre la importancia de las prácticas educativas mediante la extensión universitaria, con intercambio colectivo de conocimientos, contribuyendo a la construcción personal y profesional de todos los participantes.

DESCRIPTORES: Salud del Anciano; Educación en Salud; Atención Primaria de Salud; Enfermería; Relaciones Comunidad-Institución.

RECEBIDO EM: 31/10/2020 APROVADO EM: 10/11/2020 


\title{
Ana Claudia Torres de Medeiros
}

Enfermeira. Docente da Unidade Acadêmica de Enfermagem da Universidade Federal de Campina Grande (UAENF/UFCG). Doutora em Enfermagem pela Universidade Federal da Paraíba (UFPB).

ORCID: 0000-0002-3695-9745

\section{Natally Calixto Lucena}

Enfermeira pela UFCG.

ORCID: 0000-0002-3619-1545

\author{
Dhébora Rhanny Ribeiro Escorel Barros \\ Enfermeira pela UFCG. \\ ORCID: 0000-0001-9717-9905
}

\section{Mayse Cristelle de Sales Mélo}

Enfermeira pela UFCG.

ORCID: 0000-0002-9402-0752

\author{
Heloísa Souto Policarpo Araújo \\ Enfermeira pela UFCG. \\ ORCID: 0000-0001-6041-0595
}

\section{Kleane Maria da Fonseca Azevedo Araújo}

Enfermeira. Docente da UAENF/UFCG. Doutora em Enfermagem pela Universidade Federal de Minas Gerais (UFMG). ORCID: 0000-0001-8033-7305

\section{INTRODUÇÃO}

A diminuição da taxa de fecundidade bem como o aumento da expectativa de vida da população brasileira traz uma nova organização social, um verdadeiro desafio para a saúde pública. As projeções apontam que para o ano de 2050 os idosos representem aproximadamente $28 \%$ da população brasileira $^{(1)}$. Estas modificações, por sua vez, têm implicado importantes mudanças também no perfil epidemiológico da população, com alterações relevantes nos indicadores de morbimortalidade. Com isso, foram necessárias políticas públicas que garantissem a prevenção e a promoção de saúde para este público, como a legislação brasileira que assegura determinados direitos para a população de 60 anos ou mais de idade ${ }^{(2)}$.

Entendendo que a Política Nacional de Atenção Básica (PNAB) descreve a Atenção Básica como o primeiro nível de atenção em saúde, caracterizada por um conjunto de ações de saúde, no âmbito individual e coletivo objetivando desenvolver uma atenção integral que impacte positivamente na situação de saúde das coletividades ${ }^{(3)}$. No entanto, a assistência à saúde da pessoa idosa envolve alguns desafios, principalmente no que tange ao atual modelo de atenção à saúde, insatisfatório para atender as demandas da população idosa, uma vez que, em sua maioria tem como base ações curativistas, voltadas ao modelo biomédico ${ }^{(4)}$. Nesse sentido, é de extrema importância desenvolver estratégias de saúde específicas aos idosos, que assistam de maneira holística às suas necessidades, para além dos aspectos biológicos ${ }^{(4)}$. Em consonância com isso, a educação em saúde surge como uma importante ferramenta para mudança de paradigmas no atual modelo de atenção, apontando para um conceito ampliado em saúde, que envolve esferas ambientais, psicológicas, biológicas, interpessoais, econômicas, sociais etc.

É preciso encarar a educação em saúde como ação complexa, prática social com potência para desenvolver a reflexão e a consciência crítica das pessoas sobre seus problemas em saúde ${ }^{(5)}$. É considerada uma intervenção não farmacológica efetiva para idosos, pois proporciona uma melhor compreensão das necessidades humanas e se destaca como promoção da saúde em relação à conscientização sobre responsabili- dades e direitos relacionados à saúde ${ }^{(6)}$. $\mathrm{O}$ que foi possível realizar por meio do Programa Institucional de Bolsas de Extensão da Universidade Federal de Campina Grande (PROBEX-UFCG).

Conforme Resolução n ${ }^{\circ}$ 04/2009, sua responsabilidade é contribuir para a formação profissional dos graduandos da UFCG, a partir do envolvimento em situações concretas de ensino-pesquisa viabilizadas pelas atividades de extensão além de viabilizar a relação transformadora entre a universidade e a sociedade, priorizando as demandas de relevância social, com o intuito de melhorar as condições de vida das comunidades beneficiadas ${ }^{(7)}$.

É importante a discussão em relação ao envelhecimento e todas as alterações advindas com esse processo para que assim, possa promover uma maior longevidade com qualidade de vida principalmente através de ações educativas importante ferramenta para promover o autocuidado, incentivar e ampliar a autonomia favorecendo a independência do idoso.

Desse modo, o estudo tem como objetivo relatar a experiência de ações educativas em saúde com idosos da atenção primária à saúde. 


\section{METODOLOGIA}

Trata-se de um estudo qualitativo, descritivo, tipo relato de experiência, realizado em uma Unidade Básica de Saúde (UBS) localizada no município de Campina Grande-PB. Esse tipo de estudo consiste em uma experiência pertencente ao domínio social, fazendo parte das experiências humanas, devendo conter impressões observadas ${ }^{(8)}$.

A vivência ocorreu por meio do Projeto de Extensão, vinculado à Pró-Reitora de Pesquisa e Extensão (PROPEX), intitulado "Educação em saúde com idosos na atenção primária à saúde" desenvolvido durante o período de maio a dezembro do ano de 2018 por 10 alunos, sendo 8 do curso de Enfermagem e 2 do curso de Medicina, com a supervisão da coordenadora do projeto. Contou com a participação de 15 idosos da referida comunidade.

Inicialmente realizou-se uma reunião dos participantes do projeto com a equipe da UBS para apresentação do projeto e estabelecimento de metas. Em seguida, foram realizadas visitas domiciliares para convidar os usuários, de ambos os sexos, com idade igual ou superior a 60 anos e foi marcada a data e horário do primeiro encontro para o desenvolvimento de ações educativas. Os encontros aconteceram quinzenalmente no auditório da UBS e na Sociedade dos Amigos Bairros (SAB), localizada vizinho à Unidade.

Para o desenvolvimento das ações educativas, utilizou-se como estratégia a implementação da atual versão da Caderneta de Saúde do Idoso, preconizada pelo Ministério da Saúde e o desenvolvimento das temáticas apresentadas nela, além de outras conforme a necessidade e anseios do público-alvo. Foram realizados registros das atividades em um diário de campo. Ressalta-se que todas as ações foram planejadas e discutidas pelos participantes e que ao final de cada uma delas foi realizada a avaliação contemplando os aspectos positivos e pontos a serem melhorados.

A análise de dados foi de forma subjetiva, descritas de acordo com cada temática abordada durante as ações educativas rea- lizadas. Não foi necessária a submissão no Comitê de Ética e Pesquisa por se tratar de um relato de experiência. As atividades realizadas tiveram o intuito exclusivamente de educação e ensino, sem finalidade de pesquisa científica. No entanto, foram respeitados os preceitos éticos da Resolução $n^{\circ} 466 / 12^{(9)}$.

Com o

desenvolvimento

de algumas doenças

mais comuns na

velhice, tal como

a diabetes, existe

a possibilidade

da perda da

sensibilidade

principalmente nos

membros inferiores

denominada

de neuropatia

periférica...

\section{RELATO DE EXPERIÊNCIA}

Durante as ações, realizou-se uma abordagem inicial com todos os idosos presentes com aferição de pressão arterial e o registro na Caderneta de Saúde da Pessoa Idosa, em seguida foi trabalhada a temática planejada, conforme os temas da referi- da Caderneta ou de acordo com a temática sugerida pelos idosos. As ações foram realizadas de forma a facilitar a compreensão do público-alvo por meio de oficinas, palestras, rodas de conversa e jogos.

Mediante os oito meses de atuação do projeto, com a participação de cerca de 15 idosos, foram executadas 10 ações desenvolvidas pela professora orientadora do projeto, pelos estudantes dos cursos de Enfermagem e Medicina integrantes do projeto, alunos convidados do Programa de Educação Tutorial de Fitoterapia (PET- Fitoterapia) do Centro de Ciências Biológicas e da Saúde (CCBS/UFCG) e profissionais do Núcleo de Apoio à Saúde da Família (NASF).

Como ação inicial do projeto, foi realizada a apresentação dele para os idosos. Começando com o acolhimento por meio de uma dinâmica de interação entre os alunos e os idosos falando o nome e alguma característica pessoal. Através do diálogo entre duas alunas do projeto foi apresentada a Caderneta de Saúde da Pessoa Idosa, sua função, importância e objetivos. Em seguida foi realizada a distribuição e o preenchimento inicial dela.

Conforme sugestão da Caderneta de Saúde da Pessoa Idosa, a segunda ação educativa realizada teve como temática "Atividade física" com o objetivo de estimular a prática de atividades que proporcionam mais disposição, autonomia, relações interpessoais, diminui o risco de doenças cardíacas, diabetes e depressão. Foi desenvolvida com a participação de um professor de Educação Física quando foram realizados exercícios simples que podem ser realizados até mesmo em casa com a estimulação funcional com o intuito de motivar a sua prática.

Com o desenvolvimento de algumas doenças mais comuns na velhice, tal como a diabetes, existe a possibilidade da perda da sensibilidade principalmente nos membros inferiores denominada de neuropatia periférica, para isso foi ministrada a oficina "Cuidado com os Pés" abordando as principais orientaçôes acerca da maneira correta do corte das unhas, hidratação da pele e o tipo de calçado adequado. $\mathrm{Na}$ 
ocasião foram levados materiais como tesoura, hidratantes, lixa de unha e imagens impressas para facilitar a compreensão do conteúdo demonstrado durante a oficina.

Conforme sugestão da Caderneta de Saúde da Pessoa Idosa o tema "Alimentação saudável" foi discutido pela nutricionista a respeito dos melhores e mais indicados alimentos a serem consumidos no dia a dia. Durante a explanação do tema, os idosos levantavam uma placa na cor verde caso concordasse com a afirmativa citada ou uma placa vermelha indicando que não concordava, e deste modo foram sanadas as dúvidas e realizadas orientações.

Sabe-se que a fragilidade dos idosos aliada a fatores extrínsecos, tais como má iluminação, piso escorregadio, fazem com que as quedas tenham consequências significativas na saúde física e psicológica dos $\operatorname{mesmos}^{(10)}$. Com a ajuda da fisioterapeuta do NASF foi proporcionada a oficina sobre "Prevenção de quedas" com as principais orientações para se evitar quedas principalmente no ambiente doméstico.

Realizou-se uma roda de conversa com a temática "Alterações do corpo com o processo de envelhecimento" importante discussão em relação ao envelhecimento e todas as alterações advindas com esse processo seja ela física, psicológica ou social(11). A oficina foi guiada por meio de fotos impressas de algumas dessas alterações. Os idosos relataram as alterações sentidas e quais as estratégias utilizadas para enfrentá-las.

Sabe-se que no mês de outubro, após entrar em vigência o Estatuto do Idoso, o qual assegura os direitos das pessoas com idade igual ou superior a 60 anos e a Lei No 11.433/06 ficou instituído o dia nacional do idoso comemorado no dia $1^{\circ}$ de outubro $^{(12)}$. Fazendo alusão a essa data, foi proporcionada uma comemoração para os participantes do projeto.

Enfatiza-se também que durante esse mês realiza-se a Campanha nacional de prevenção ao câncer de mama com o intuito de alertar sobre prevenção e diagnóstico precoce. Para tanto, foi desenvolvida a ação com o tema "Orientações para o autoexame das mamas" sensibilizando as pessoas idosas sobre a importância da detecção e diagnóstico precoce. Utilizou-se um manequim com as mamas facilitando a demonstração de como deve ser realizado o autoexame e imagens impressas com as principais alterações das mamas.

Discutiu-se também, com a colaboração dos alunos do PET-Fitoterapia, o uso de plantas medicinais no cotidiano dos idosos. Foi solicitado que os idosos apresentassem os fitoterápicos e plantas medicinais que geralmente fazem uso, sendo discutido os efeitos, indicações e modo de preparo dos mais utilizados dirimindo-se as dúvidas. Foi distribuído um panfleto com orientações gerais sobre o uso de fitoterápicos. E para o feedback em relação à temática, os alunos utilizaram um jogo da memória que além de divertir os idosos facilitou a aprendizagem.

Durante as ações do projeto, viu-se a importância de falar sobre "Sexualidade e infecções sexualmente transmissíveis (ISTs)" que continuam presentes nas pessoas independentemente da idade. Explicou-se que, com o passar dos anos algumas condições podem interferir na vida sexual, e que a idade não dará proteção contra as ISTs. Destacou-se a importância do uso de preservativo, como uma das melhores formas de prevenção. Além disso, foram realizados os testes para sífilis, HIV/Aids e hepatite $\mathrm{C}$, e entregues preservativos. Ressalta-se que quanto antes forem identificados, mais cedo pode-se iniciar o tratamento e evitar a transmissão(13).

Os idosos sempre se mostraram satisfeitos com a realização das ações, com relatos de que o projeto era muito bom e acrescentava muito na vida deles, o qual se confirmou com a assiduidade nas ações que objetivou promover uma troca de experiência entre os idosos e os alunos para o bem-estar e melhor qualidade de vida para aquela comunidade beneficiada pelo projeto de extensão.

\section{DISCUSSÃO}

Chegar à velhice, que antes era privilégio de poucos, passou a ser acontecimento mesmo nos países mais pobres. Mesmo sendo uma conquista maior do século XX, isso se transformou, no grande desafio para o século atual. $\mathrm{O}$ envelhecimento da população não basta por si só. Sabe-se que o estilo de vida adotado pelo idoso afeta diretamente em seu processo de envelhecimento. A velhice é conhecida como heterogênea pois, algumas pessoas envelhecem com boa qualidade de vida e saúde, com poucas enfermidades, já outros vivenciam inatividade, fadiga, comorbidades que afetam na sua qualidade de vida ${ }^{(14)}$.

Portanto, é importante que se consiga agregar qualidade aos anos adicionais de vida ${ }^{(15)}$. Por isso, a importância de trabalhar a promoção e prevenção de doenças o que irá proporcionar idosos ativos e saudáveis. Nessa perspectiva, o Ministério da Saúde lançou a atual Caderneta de Saúde da Pessoa Idosa, como um instrumento estratégico de qualificação da atenção ao idoso, que objetiva organizar o processo de trabalho das equipes de saúde e a otimização da avaliação integral da saúde da pessoa idosa, identificando suas principais vulnerabilidades e oferecendo orientações de autocuidado ${ }^{(13)}$.

É um instrumento proposto para auxiliar no bom manejo da saúde da pessoa idosa, sendo usada tanto pelas equipes de saúde, quanto pelos idosos, seus familiares e cuidadores ${ }^{(13)}$. Apresenta dados pessoais, uma avaliação geral da pessoa idosa, calendário de vacina e avaliação de saúde bucal, espaço para agendamento de consultas e resultados de exames e hábitos de vida, informações importantes para elaboração do projeto terapêutico.

Sabe-se que a educação em saúde deve ser baseada na construção de relacionamentos e ambientes democráticos que ofereçam troca de conhecimento com o compromisso da humanização, o qual inclui o respeito às características geográficas, sociais, políticas e culturais da pessoa, família e comunidade usando preferencialmente métodos de aprendizagem ativos ${ }^{(6)}$.

Compreende-se como importante a atividade educativa lúdica e ativa, pois elas estimulam a compreensão de temas, de maneira prazerosa, gerando reflexão sobre os conhecimentos adquiridos e criando 
relações entre conhecimento produzido de forma recreativa e vivenciada, incluindo aspectos comportamentais individuais e coletivos ${ }^{(6)}$. Sendo assim, práticas fundamentadas na produção de um cuidado integral constituídas em consonância com os princípios da universalidade e da equidade, na composição do Sistema Único de Saúde (SUS) ${ }^{(16)}$.

Dessa forma, é de fundamental importância incentivar a prática de ações educa- tivas para os idosos, cuja participação ativa contribui no fortalecimento de vínculos, interação interpessoal, melhoria no desempenho das atividades de vida diária, bem-estar emocional, na adesão às atividades propostas, funcionalidade e consequentemente na sua qualidade de vida.

\section{CONCLUSÃO}

A educação em saúde é considerada uma forma de cuidado humanizado e holístico proporcionando uma construção contínua e coletiva do conhecimento por meio das atividades realizadas em grupo. Espera-se que este relato sensibilize a comunidade acadêmica quanto à importância da extensão universitária como facilitadora e priorizando as demandas de relevância social, para melhorar as condiçóes de vida das comunidades, sendo uma ponte para potencializar as políticas públicas. -

\section{REFERÊNCIAS}

1. Brasil. Instituto Brasileiro de Geografia e Estatística (IBGE). Projeção da população do Brasil e Unidades da Federação por sexo e idade para o período 2010-2060: revisão 2018. [Internet]. Rio de Janeiro. 2018 [cited 2020 Nov 6]. Avaliable from: https:// www.ibge.gov.br/estatisticas/sociais/populacao/9109-projecao-da-populacao.html?=\&t=resultados

2. Ministério da Saúde. Portaria $n^{\circ} 2.528$ de 19 de outubro de 2006. Aprova a Política Nacional de Saúde da Pessoa Idosa. [Internet]. Brasilia, DF, 2006. [cited 2020 Oct 29]. Avaliable from: https://bvsms.saude.gov.br/bvs/saudelegis/gm/2006/ prt2528_19_10_2006.html

3. Brasil. Ministério da Saúde. Departamento de Atenção Básica. [Internet]. Brasília, DF, 2018. Nov. [cited 2019 Jun 23] Avaliable from: http://aps.saude.gov.br/smp/smpoquee

4. Castro APR, Vidal ECF, Saraiva ARB, Arnaldo SM, Borges AMM, Almeida MI. Promoção da saúde da pessoa idosa: ações realizadas na atenção primária à saúde. Rev. bras. geriatr. gerontol. [Internet]. 2018 Apr [cited 2020 Nov 06]; 21(2): 155-163. Avaliable from: http://dx.doi.org/10.1590/1981-22562018021.170133

5. Borba AKOT, Arruda IKG, Marques AP de O, Leal MCC, Diniz AS. Conhecimento sobre o diabetes e atitude para o autocuidado de idosos na atenção primária à saúde. Ciênc. saúde coletiva [Internet]. 2019 Jan [cited 2019 Jun 23] ; 24(1):125-136. Avaliable from: http://dx.doi.org/10.1590/141381232018241.35052016

6. Casemiro FG, Quirino DM, Diniz MAA, Rodrigues RAP, Pavarini SCI, Gratão ACM. Efeitos da educação em saúde em idosos com comprometimento cognitivo leve. Rev. Bras. Enferm. [Internet]. 2018 [cited 2019 Jun 23]; 71(Suppl 2): 801-810. Avaliable from: http://dx.doi.org/10.1590/0034-7167-2017-0032

7. Brasil. Universidade Federal de Campina Grande. Resolução $n^{\circ}$ 04/2009, de 15 de abril de 2009. Fixa normas para o programa institucional de bolsas de Extensão da UFCG - Probex conselho universitário câmara superior de pesquisa e extensão [Internet]. Campina Grande-PB. 2009. [cited 2019 Jun 23]. Avaliable from: http://www.extensao.ufcg.edu.br/arquivos/phocadownload/PROBEX/res_15042009.pdf

8. Lopes MVO. Sobre estudos de casos e relatos de experiências. Rev. da Rede de Enfermagem do Nordeste [Internet]. 2015 [cit- ed 2019 Jun 23];13. Avaliable from: http://www.periodicos.ufc. br/rene/article/view/4019

9. Brasil. Resolução no466 de 12 de dezembro de 2012. Dispõe sobre diretrizes e normas regulamentadoras de pesquisas envolvendo seres humanos [Internet] 2012 [cited 2019 Jun 27]. Avaliable from: http://conselho.saude.gov.br/ultimas_noticias/2013/06_jun_14_publicada_resolucao.html

10. Abreu DROM, Novaes ES, Oliveira RR de, Mathias TAF, Marcon SS. Internação e mortalidade por quedas em idosos no Brasil: análise de tendência. Ciênc. saúde coletiva [Internet]. 2018 Apr [cited 2019 Jun 23]; 23(4): 1131-1141. Avaliable from: http://dx.doi.org/10.1590/1413-81232018234.09962016

11. Sublime. Saúde. Processo de envelhecimento humano: Como envelhecer com saúde, 2018. [cited 2019 Jun 23] Avaliable from: https://saudesublime.com/processo-de-envelhecimento/

12. Brasil. Decreto Lei № 11.433 de 28 de dezembro de 2006. Dispõe sobre o Dia Nacional do Idoso, Brasília, DF, Dez 2006. [cited 2019 Jun 23]. Avaliable from: http://www.planalto.gov.br/ ccivil_03/_Ato2004-2006/2006/Lei/L11433.htm

13. Brasil. Ministério da Saúde. Secretaria de Atenção à Saúde. Departamento de Ações Programáticas Estratégicas. Caderneta de saúde da pessoa idosa. 5a . Ed. - Brasília: Ministério da Saúde, 2018 [cited 2019 Jun 27] Avaliable from: http://bvsms.saude. gov.br/bvs/publicacoes/caderneta_saude_pessoa_idosa.pdf

14. Garbaccio JL, Tonaco LAB, Estêvão WG, Barcelos BJ. Envelhecimento e qualidade de vida de idosos em áreas rurais. Rev Bras Enferm [Internet]. 2018 [cited 2019 Jun 24]; v.71(suppl 2): p.724-32. Avaliable from: http://dx.doi.org/10.1590/00347167-2017-0149

15. Veras RP, Oliveira M. Envelhecer no Brasil: a construção de um modelo de cuidado. Ciênc. Saúde coletiva [Internet]. 2018 Jun [cited 2019 Jun 27]; 23(6): 1929-1936. Avaliable from: http://dx.doi.org/10.1590/1413-81232018236.04722018

16. Silva, SS da, Assis MMA, Santos AM dos. Enfermeira como protagonista do gerenciamento do cuidado na estratégia saúde da família: diferentes olhares analisadores. Texto contexto - enferm. [Internet]. 2017 [cited 2019 Jun 24] ; 26(3): e1090016. Avaliable from: http://dx.doi.org/10.1590/010407072017001090016 